\title{
Male-Female Interactions and the Evolution of Postmating Prezygotic Reproductive Isolation among Species of the Virilis Subgroup
}

\author{
Nada Sagga and Alberto Civetta \\ Department of Biology, University of Winnipeg, 515 Portage Avenue, Winnipeg, MB, Canada R3B 2E9 \\ Correspondence should be addressed to Alberto Civetta, a.civetta@uwinnipeg.ca \\ Received 25 October 2010; Accepted 3 February 2011 \\ Academic Editor: Jeremy Marshall
}

Copyright (C) 2011 N. Sagga and A. Civetta. This is an open access article distributed under the Creative Commons Attribution License, which permits unrestricted use, distribution, and reproduction in any medium, provided the original work is properly cited.

Reproductive isolation reduces breeding between species. Traditionally, prezygotic and postzygotic barriers to reproduction have been broadly studied, but in recent years, attention has been brought to the existence of barriers that act after copulation but before fertilization. Here, we show that when $D$. virilis females from different geographic locations mate with $D$. novamexicana males, egg laying is normal, but fertilization rates are severely reduced, despite normal rates of sperm transfer. This reduction in fertilization is probably due to lower retention of heterospecific sperm in female storage organs one-to-two days after copulation. An inspection of egg hatchability in crosses between females and males from other virilis subgroup species reveals that isolation due to poor egg hatchability likely evolved during the diversification of $D$. virilis/D. lummei from species of the novamexicana-americana clade. Interestingly, the number of eggs laid by $D$. virilis females in heterospecific crosses was not different from the numbers of eggs laid in conspecific crosses, suggesting that females exert some form of cryptic control over the heterospecific ejaculate and that future studies should focus on how female and female-sperm interactions contribute to the loss or active exclusion of heterospecific sperm from storage.

\section{Introduction}

Species can be reproductively isolated by a variety of mechanisms that as a whole reduce gene flow between them. These mechanisms can be broadly classified into prezygotic and postzygotic isolating barriers [1]. A large number of studies have drawn attention to isolating barriers in which gene flow is reduced after mating but prior to zygote formation [210]. While some of these barriers are competitive, others are a consequence of male-female incompatibilities and are noncompetitive, disrupting the sperm's capacity to reach and/or fertilize an egg. In Drosophila, noncompetitive isolating mechanisms have been well described. Sperm transfer from $D$. arizonae males to $D$. mojavensis females generates an insemination reaction in which a large mass forms in the uterus that obstructs ovulation and ultimately fertilization [9]. In crosses between D. simulans females and D. sechellia males, fewer sperm are transferred, and egg-laying and hatchability are reduced [5]. Sperm are also depleted more quickly in crosses between D. santomea and D. yakuba [10].

The virilis subgroup consists of five species: $D$. virilis, $D$. lummei, D. novamexicana, $D$. americana americana, and $D$. americana texana. There is evidence for both premating and postmating isolation among these five species. Drosophila virilis females have the highest crossability with all heterospecific males of the subgroup, whereas $D$. virilis males show the strongest courtship discrimination against all heterospecific females with the result that very low numbers of hybrids are produced [11]. A recent study has shown that when $D$. novamexicana females are exposed to $D$. virilis males for up to two weeks, only $14 \%$ of females produce progeny. Moreover, $D$. virilis males are able to recognize heterospecific females at the first stage of courtship (tapping), indicating strong premating isolation [12]. For the reciprocal cross between $D$. virilis females and $D$. novamexicana males, there is evidence of strong postzygotic isolation with $7 \%$ hybrid 
male fertility [13]. Drosophila virilis females mated with $D$. lummei males produce $95 \%$ fertile hybrid males, but $47 \%$ of the hybridizations die before becoming pupae, and 25\% fail to emerge from their case [14]. When $D$. virilis females mate with $D$. a. texana males, one-third of the hybrid male progeny is sterile $[13,15]$.

The occurrence of postmating prezygotic isolation (PPI) barriers among species of the virilis subgroup has also been documented. For example, early studies showed that $D$. virilis females mated to heterospecific males produced very few offspring. These studies suggested, although qualitatively, that the low production of progeny was due to sperm immobility (mortality) in the seminal receptacle of the females' reproductive tract for crosses between $D$. virilis females and $D$. a. americana or $D$. a. texana males but not for crosses involving D. novamexicana males [17, page 489]. A recent study has shown a low rate of egg hatchability when $D$. virilis females are mated to $D$. a. americana males with most of the laid eggs not being fertilized [16].

Drosophila virilis females mated to $D$. novamexicana males have previously shown to hatch a low proportion of eggs after mating, but the reasons for such low egg hatchability remain unclear [17]. Here, we explore mechanisms of reproductive isolation between $D$. virilis females and heterospecific males by mainly, but not exclusively, focusing on crosses with $D$. novamexicana males. We first test strains of different geographic origin to confirm that prior reports of low hatchability for this cross are not strain dependant. Secondly, we use outbred populations to rule out the possibility that laboratory inbreeding might create or exacerbate some of the isolation phenotypes detected. Thirdly, we show that females hatch low proportion of eggs after a heterospecific cross but lay similar numbers of eggs in heterospecific and conspecific crosses. Fourthly, within the virilis subgroup, we show that the only cross for which isolation between species due to low hatching of eggs has not evolved is that between $D$. virilis females and D. lummei males. Finally, using outbred D. virilis females and outbred D. novamexicana males, we show that the low hatchability of eggs is not a consequence of embryo mortality but due to problems associated with female storage of heterospecific sperm.

\section{Materials and Methods}

2.1. Drosophila Species and Maintenance. Five geographically diverse strains of $D$. virilis (Argentina 15010-1051.49, California 15010-1051.00, Japan 15010-1051.09, Mexico 150101051.48, and Russia 15010-1051.52), two D. novamexicana strains (New Mexico 1301.08 and Utah 1301.08), a strain of D. lummei (Japan 1011.08), and a strain of D. americana texana (wild type 1041.16) were obtained from the San Diego Drosophila Stock Center. The number of eggs laid and the proportion of eggs hatched can be affected by inbreeding in laboratory strains of Drosophila, so we also established outbred populations of $D$. virilis and $D$. novamexicana by mixing equal numbers of individuals from the different strains in a population cage for five generations. Flies were reared in round-bottom bottles $(64 \times 130 \mathrm{~mm})$ containing standard cornmeal-yeast-agar-molasses (CYAM) medium. Bottles were kept in a $12: 12$ light-dark cycle and at $18^{\circ} \mathrm{C}$ $-20^{\circ} \mathrm{C}$. For stock maintenance, flies were allowed to freely mate and laid progeny in fresh media, the adults were discarded after 18 days, and a new generation of newly emerged adults were transferred to fresh media.

\subsection{Setting Up Crosses for Experimental Testing. Crosses were} performed between $D$. virilis females and $D$. novamexicana males as well as D. lummei and D. a. texana males. Reciprocal crosses among species were also tested with the exception, due to strong premating isolation [12], of crosses using D. virilis males. Conspecific crosses were used as controls. Bottles from each species stock were emptied and inspected daily for new adult emergences. Newly emerged flies were lightly anesthetized using $\mathrm{CO}_{2}$ gas. Virgin females and males were separated by sex and placed in cylindrical vials $(28.5 \times 95 \mathrm{~mm})$ containing CYAM medium. Males and females were aged for 10 days before use to ensure sexual maturity [18] and crosses were set up with one male and one female. Counts were obtained of eggs laid by females and the proportion of eggs hatched. For crosses between outbred $D$. virilis and D. novamexicana, we also estimated the proportion of fertilized eggs, and sperm fate was tracked within females.

2.3. Egg Hatchability. Each conspecific and heterospecific pair was placed in an egg-laying chamber made using a polystyrene Petri dish $(60 \times 15 \mathrm{~mm})$ containing fresh CYAM medium attached to a $100 \mathrm{~mL}$ graduated polypropylene beaker. Every 24 hours, flies were slightly anesthetized using $\mathrm{CO}_{2}$, the Petri dish was removed, and a new dish with fresh CYAM medium was attached to the chamber. The replacement of dishes was continued for a total of five consecutive days. Eggs laid in each dish were daily counted, and 48 hours later hatched eggs were scored. Unhatched eggs can be recognized as a white compact shape, while hatched eggs appear as an empty outer chorion membrane due to larvae emergence.

2.4. Fertilization of the Eggs. Single male and female heterospecific and conspecific crosses, using $D$. virilis and $D$. novamexicana outbred strains, were set up in vials. Courtship behavior was observed until copulation occurred and for no longer than six hours. After copulation, females were transferred individually into egg-laying chambers. Females were transferred daily for five days to fresh egg-laying chambers, and the number of eggs laid was counted. 48 hours after eggs were laid, hatched eggs were scored. Unhatched eggs were collected from the media with a wooden handle dissecting pin and placed on a clean microscope slide. A drop of 1 X PBS was added to the eggs. Eggs were manually dechorionated by removing the dorsal appendage and gently pressing at the posterior pole using minute pins $(0.1 \mathrm{~mm}$ diameter). The inner vitelline membrane was removed by immersing the dechorionated eggs in a small tube containing a $1: 1$ solution of heptane and $90 \%$ methanol. The eggs 
dropped to the layer between heptane and methanol and slowly descended to the bottom of the tube when their waxy layer was lost [19]. Eggs without vitelline membrane are almost transparent and easily damaged. Intact eggs were collected by pouring the heptane-methanol solution on a small piece of dark cloth. Within one minute, the solution evaporated and the eggs were visible on the cloth surface. A couple of drops of $1 \mathrm{X}$ PBS were added on the eggs using a glass Pasteur pipette. The eggs were then gently picked using an insect pin, placed on a clean microscope slide containing 300 nM DAPI (Molecular Probes, D3571) and incubated in a dark room for 30 minutes. After incubation, the eggs were examined under a Nikon Eclipse (E400) fluorescence microscope for evidence of nuclear division (fertilization).

2.5. Tracking of Sperm. A single male and female were set up as described in the previous section. At different intervals after mating (0, 24 and 48 hours), inseminated females were transferred into vials. The vials were flash frozen by submersion in liquid nitrogen. Females were then transferred to small tubes and stored in a freezer at $-70^{\circ} \mathrm{C}$. Flies were placed in a drop of 1X PBS and each female reproductive tract was separated from the rest of the body. Sperm storage organs, uterus, pair of spermatheca, and seminal receptacle, were furthered separated, and each was placed on a fresh drop of 1X PBS on a single clean microscope slide. These slides were dried in an oven set to $60^{\circ} \mathrm{C}$ for 5 minutes, fixed in $3: 1$ methanol-glacial acetic acid for 5 minutes, and washed three times in 1X PBS [5]. Dissected tissues were stained using $300 \mathrm{nM}$ of DAPI by incubating in a dark room for 30 minutes. Slides were examined under a Nikon Eclipse (E400) fluorescence microscope, and the presence of sperm was determined.

2.6. Data Analysis. The number of eggs, laid, the proportion of hatched eggs and the proportion of eggs fertilized were compared among different crosses using a one-way analysis of variance (ANOVA). When significant differences were found among crosses, a posteriori Tukey test was performed to test which cross averages were significantly different from one another. All statistical tests were conducted in SPSS (version 12.0). Comparisons of number of females with sperm in storage were done using a $2 \times 3$ Chi-Square test.

\section{Results}

The average number of eggs laid by females mated to heterospecific males was not significantly different than the number laid by $D$. virilis females mated to conspecific males (Figure 1). Only crosses involving two $D$. virilis strains, Russia $\left(F_{2,101}=28.60 ; P<.001\right)$ and Japan $\left(F_{2,91}=22.23\right.$; $P<.001$ ), showed a significantly lower number of eggs laid than $D$. novamexicana females mated to conspecific males (Figure 1). In contrast, the proportion of eggs hatched from the heterospecific cross was consistently and significantly lower than the proportion of hatches from both of the conspecific crosses (Figure 2). Depending on the D. virilis strain used, the proportion of unhatched eggs varied between
0.84 and 0.96 (Figure 2). When using flies from the outbred populations, we found that the number of eggs laid was not different among crosses $\left(F_{2,77}=2.83 ; P=.065\right)$ (Figure 1) but that 0.94 of the eggs laid by $D$. virilis females mated with D. novamexicana males did not hatch (Figure 2).

Unhatched eggs could result from fertilized eggs that fail to develop or unfertilized eggs. We DAPI stained unhatched eggs to test for nuclear division. We counted the number of eggs hatched as fertilized and tested unhatched eggs laid from both conspecific and heterospecific crosses using the outbred populations. We found significant differences in the proportion of fertilized eggs $\left(F_{2,68}=173.42 ; P<.001\right)$ due to a significantly lower proportion of only five percent of eggs fertilized by $D$. novamexicana males that mated with $D$. virilis females (Tukey post hoc test: $P<.001$ ) (Figure 3 ). These results indicate that the vast majority of unhatched eggs in heterospecific crosses between $D$. virilis females and D. novamexicana males are the result of some form of PPI.

PPI can result from problems in sperm transfer during copulation, problems with sperm storage, or the inability of sperm to fertilize heterospecific eggs. We used the $D$. virilis and $D$. novamexicana outbred populations to test whether sperm transfer and/or storage was affected in the heterospecific cross. We did this by observing copulations and dissecting females immediately after mating (0 hours) and at two intervals of 24 and 48 hours after mating. Immediately after mating, large numbers of sperm transferred to the females were found to be located in the uterus in both conspecific and heterospecific crosses. Because of the large numbers and the fact that the sperm head of $D$. novamexicana and $D$. virilis males is a needle-like structure that sometimes only faintly stains with DAPI, we tested differences between crosses by scoring numbers of females with or without sperm in storage. There were no significant differences in numbers of females with sperm in storage immediately after mating ( 0 hours), with all females having large amounts of sperm in their uterus. At 24 hours after mating, we observed a slight decline in numbers of females with sperm in storage for the heterospecific crosses. We only found significant differences between intraand interspecific crosses for both the spermatheca and the seminal receptacle at 48 hours after mating, with a higher number of females mated to heterospecific males having no sperm in storage (spermatheca: $\chi^{2}=11.31, P=.004$; seminal receptacle: $\chi^{2}=25.23, P<.001$ ) (Figure 4 ). The most striking difference was that only 1 out of $21 D$. virilis females mated with $D$. novamexicana males had few sperm cells in the seminal receptacle at 48 hours after mating. Overall, the heterospecific cross shows a different pattern of sperm storage within the female reproductive tract than the conspecific crosses (Figure 4).

Using males from other species of the virilis subgroup, we show that the number of eggs produced by $D$. virilis females is significantly higher than the number of eggs laid by $D$. $a$. texana $\left(F_{2,48}=10.77 ; P<.001\right)$ and $D$. lummei $\left(F_{2,49}=\right.$ 3.74; $P<.031$ ) (Figure 5(a)). Interestingly, the number of eggs laid by $D$. virilis females mated to heterospecific males is not significantly different than the number of eggs laid by $D$. virilis females mated with conspecific males 


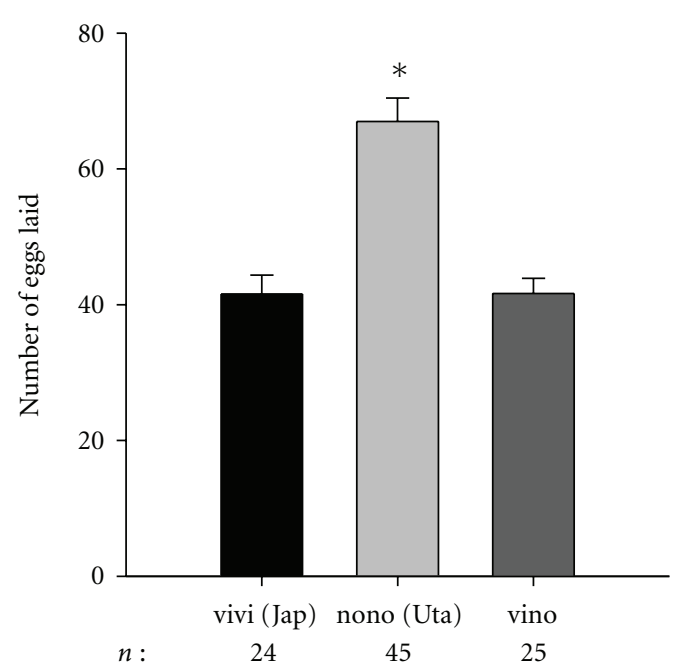

(a)

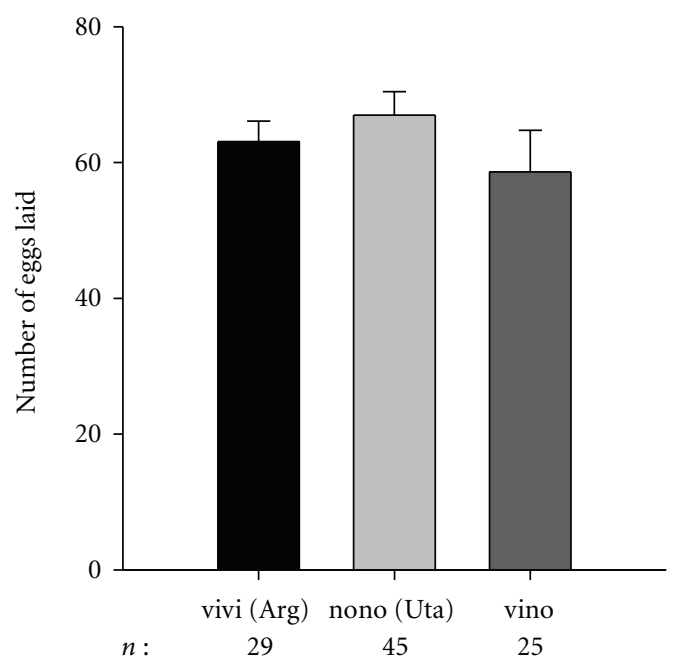

(c)

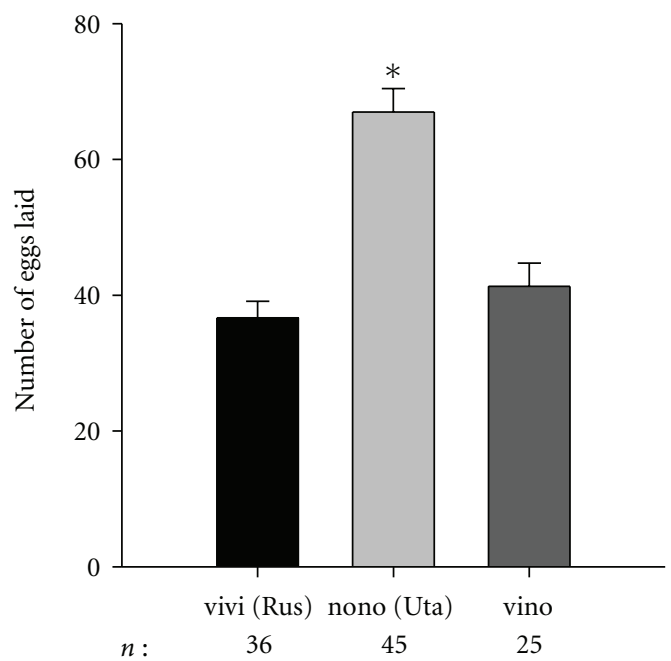

(e)

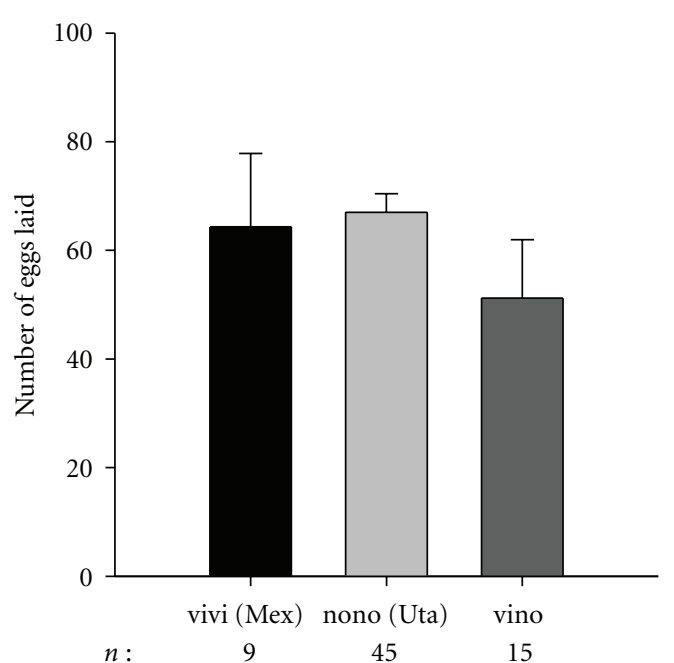

(b)

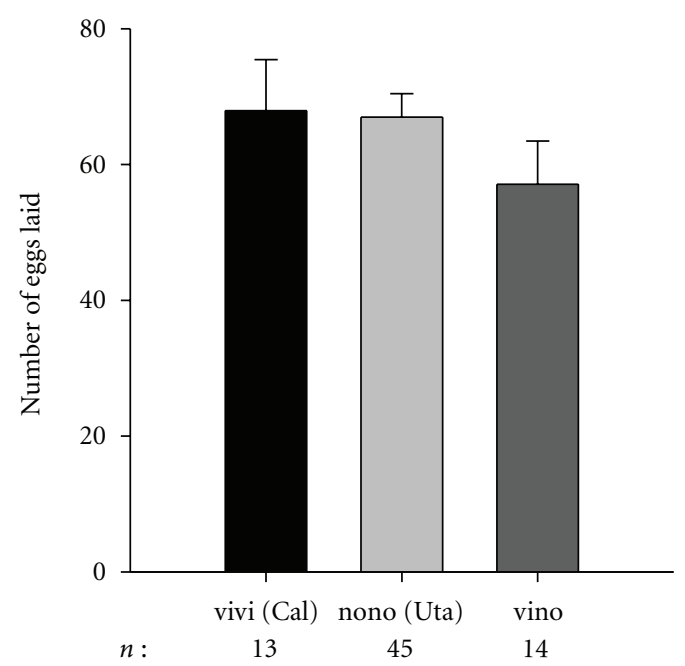

(d)

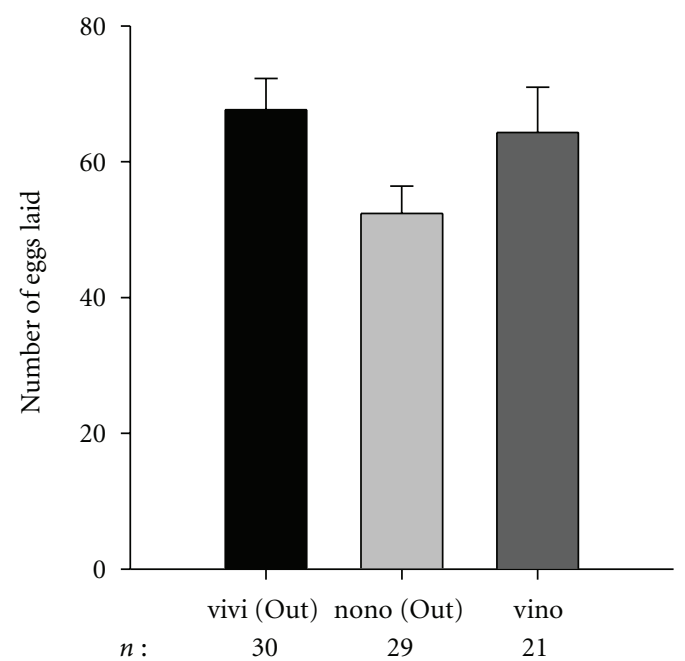

(f)

FIgURE 1: Average number of laid eggs and associated standard error. $n$ : the number of females tested. A conspecific cross is denoted as vivi or nono, for $D$. virilis and D. novamexicana, respectively, with the origin of the strain used shown in between parenthesis. Results for the heterospecific cross are labeled as vino. Asterisks above columns are used to label statistically different averages. 


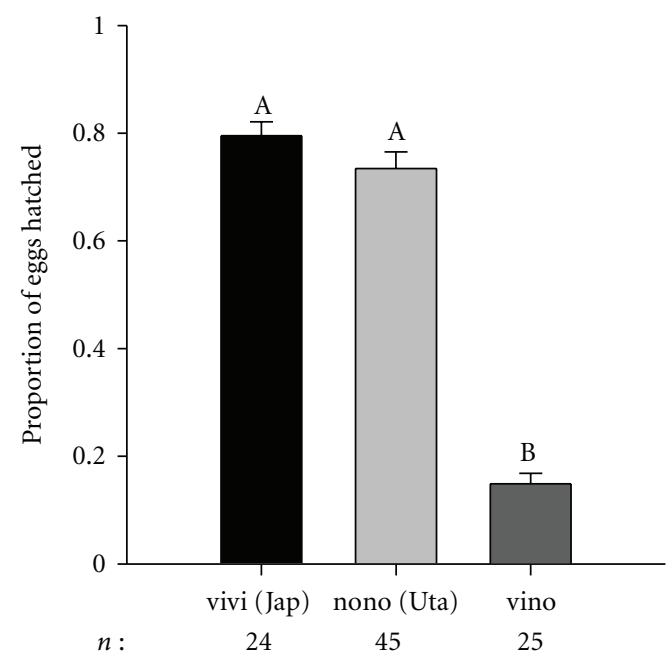

(a)

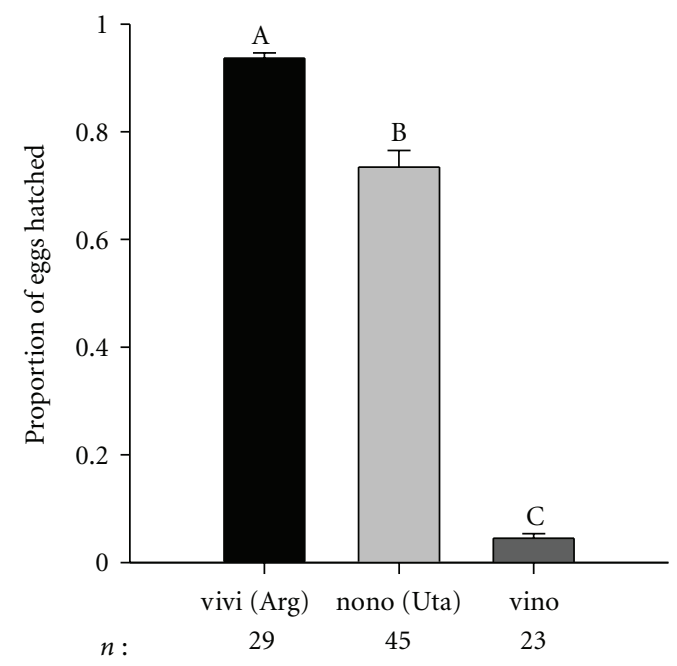

(c)

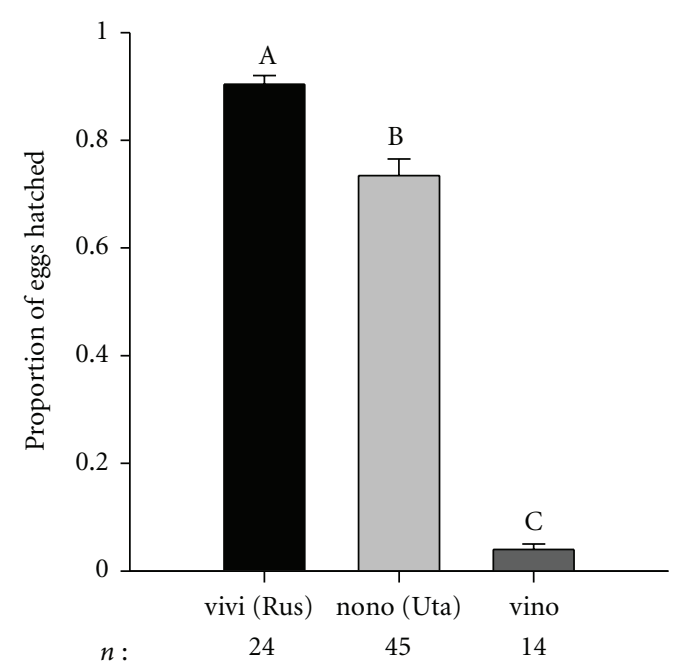

(e)

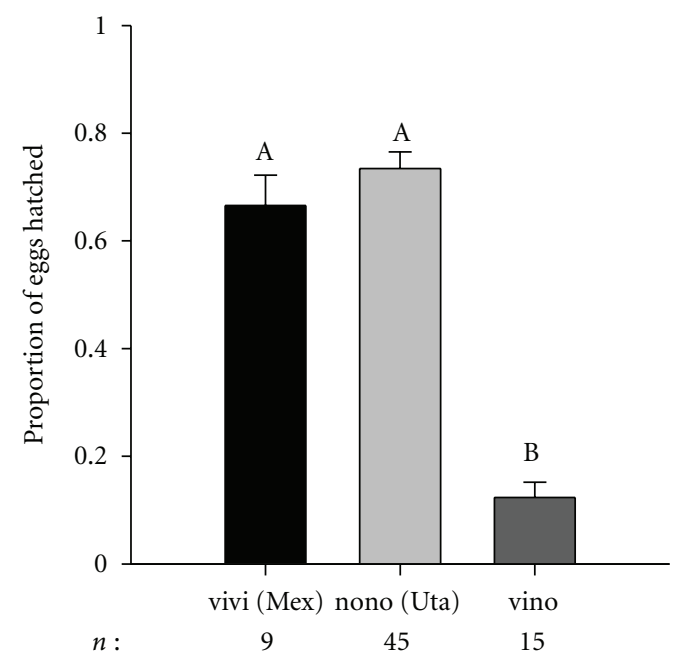

(b)

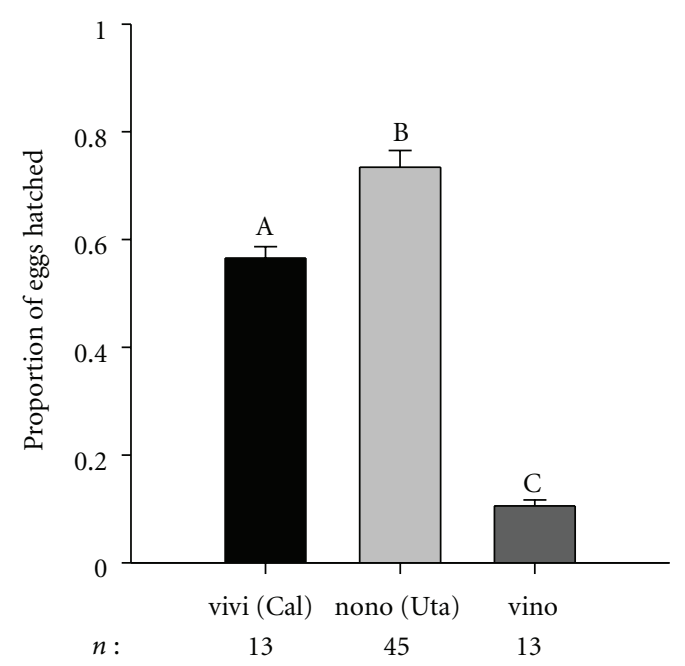

(d)

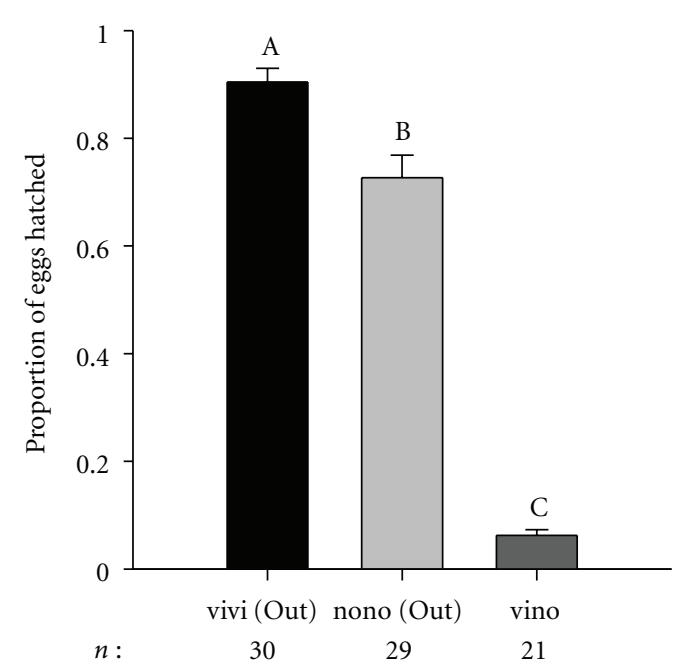

(f)

FigURE 2: Average proportion of hatched eggs and associated standard error. Labels are as in Figure 1 except that shared letters above columns indicate that averages are not statistically different. 

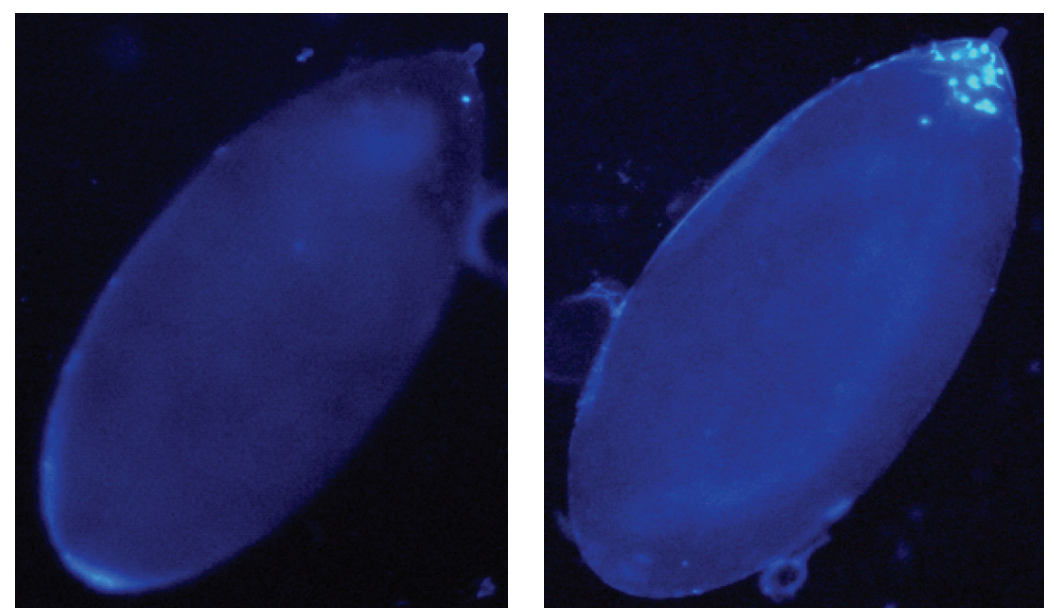

(a)

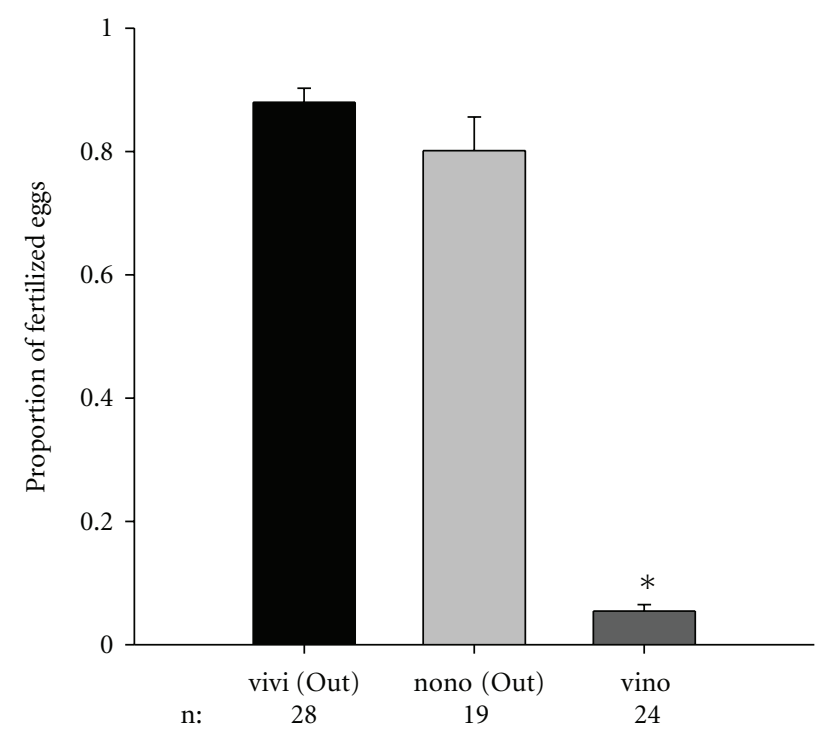

(b)

FIGURE 3: DAPI staining of eggs showing no nuclear division in an unhatched egg from a heterospecific cross (left panel) and a cluster of dividing nuclei at 2 hours after egg laying by a female mated to a conspecific male (a). Average proportion of fertilized eggs and associated standard error (b). Labels are as in Figure 1.

(Tukey post hoc test: $P=1.00 ; P=.567 ; P=.709 ; P=.944$ for $D$. virilis Japan $\times D$. novamexicana, $D$. virilis Russia $\times D$. novamexicana, $D$. virilis $\times D$. a. texana, and $D$. virilis $\times D$. lummei resp.) (Figures 1 and 5(a)). However, egg hatchability is always lower in heterospecific crosses than both conspecific crosses, with the exception of $D$. virilis females $\times D$. lummei males (Figures 2 and 5(b)).

\section{Discussion}

Studies of reproductive isolation between species of the virilis subgroup have shown extensive variation in the strength of the isolating barrier depending on the geographic origin of the strains tested (reviewed in [11]). Therefore, it is important to show that PPI between $D$. virilis females and $D$. novamexicana males is independent of the geographic origin of the strains used and thus fixed rather than polymorphic. Drosophila virilis females of different geographic origin crossed with $D$. novamexicana males hatched between $4 \%$ and $16 \%$ of eggs, in line with a prior single strain assay that found only $15 \%$ of eggs hatched within two days after mating [17, see Table 103]. The uniformity of PPI among geographically distinct $D$. virilis strains suggests that PPI with D. novamexicana is a byproduct of divergent evolution rather than reinforcement though ecological differences between the two species may have reduced any possible interactions.

Contrary to what has been found among other species of Drosophila (e.g., [10]), D. virilis females mated to heterospecific males produce numbers of eggs that are not different from the numbers produced by $D$. virilis mated to conspecific males. Numerous studies have shown the influence of accessory gland proteins (ACPs) on female oviposition in 


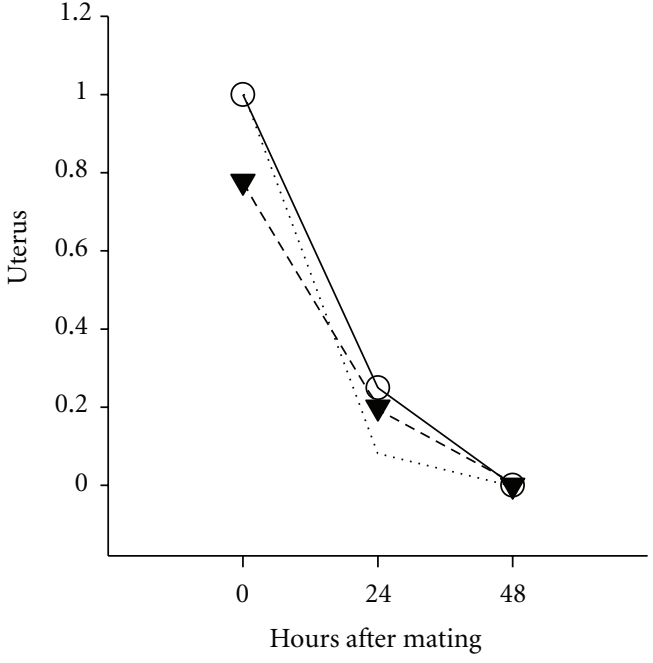

(a)

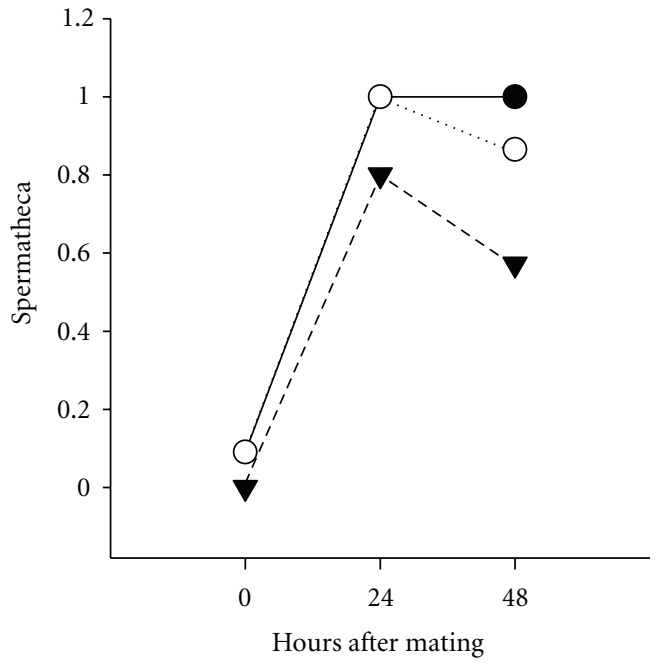

(b)

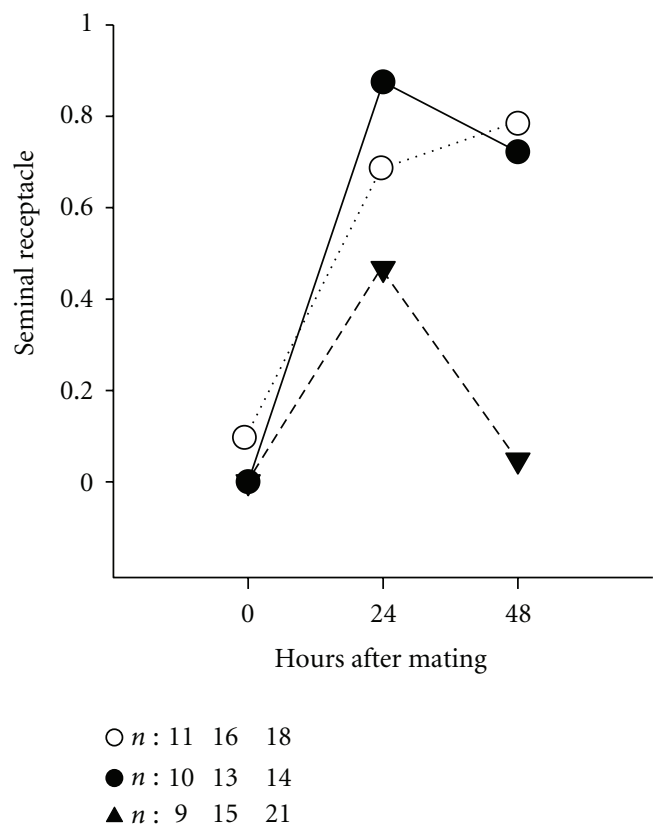

(c)

FIGURE 4: Proportion of females with sperm found in different storage organs immediately after mating ( 0 hours), at 24 hours and 48 hours after mating. White circles are $D$. novamexicana $\times D$. novamexicana, black circles are $D$. virilis $\times D$. virilis, and triangles are $D$. virilis $\times D$. novamexicana. $n$ : the number of females tested.

inseminated D. melanogaster females (reviewed in [20]). For example, both ovulin (ACP26Aa) and the sex peptide (ACP70A) have been implicated in stimulating egg production and ovulation after mating [21-24]. Males can influence female egg laying, but the egg-laying effect triggered by the ejaculate is also mediated by females' molecular counterparts (reviewed in [20]). Therefore, it is equally likely that either males of the virilis subgroup has not diverged enough in ACPs, and other ejaculate proteins content or that $D$. virilis females have retained the ability to recognize a wide variety of male-derived egg-laying stimulating signals from the ejaculate. The first possibility seems unlikely given the fast rate of evolution of ACPs in Drosophila. ACPs are known to become neofunctionalized, lose their function or simply be lost even in comparisons among closely related species of Drosophila [25-27]. However, despite the fact that current genome information (http://flybase.org/) shows no orthologs of known D. melanogaster ACPs in D. virilis, we cannot fully rule out ACPs, as we have no knowledge of divergence among these proteins within the virilis subgroup. Our results demonstrate that in every heterospecific cross involving $D$. virilis females, egg laying is determined by the female of the species. This supports the hypothesis that $D$. virilis females have retained the molecular ability to recognize 

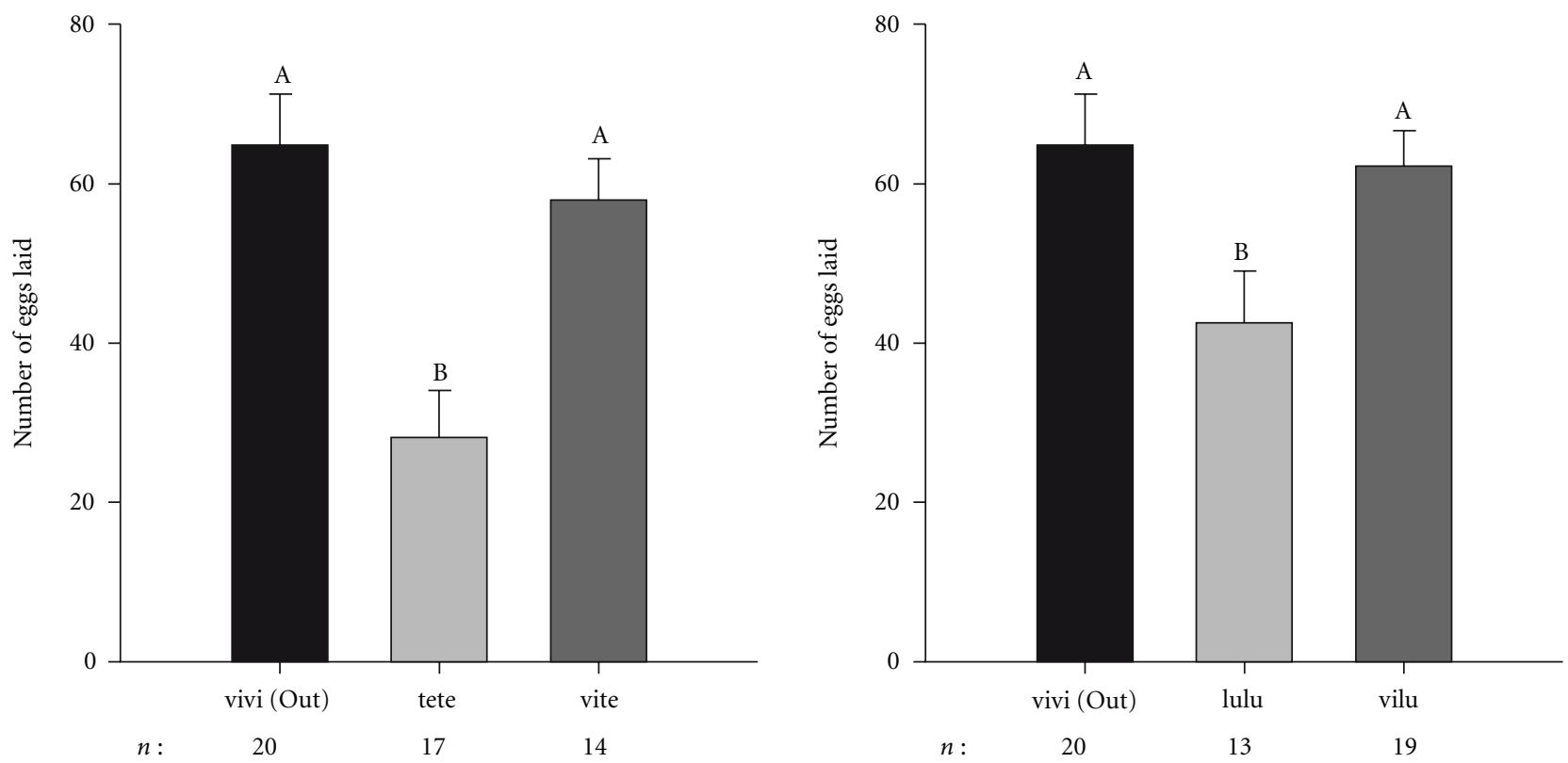

(a)
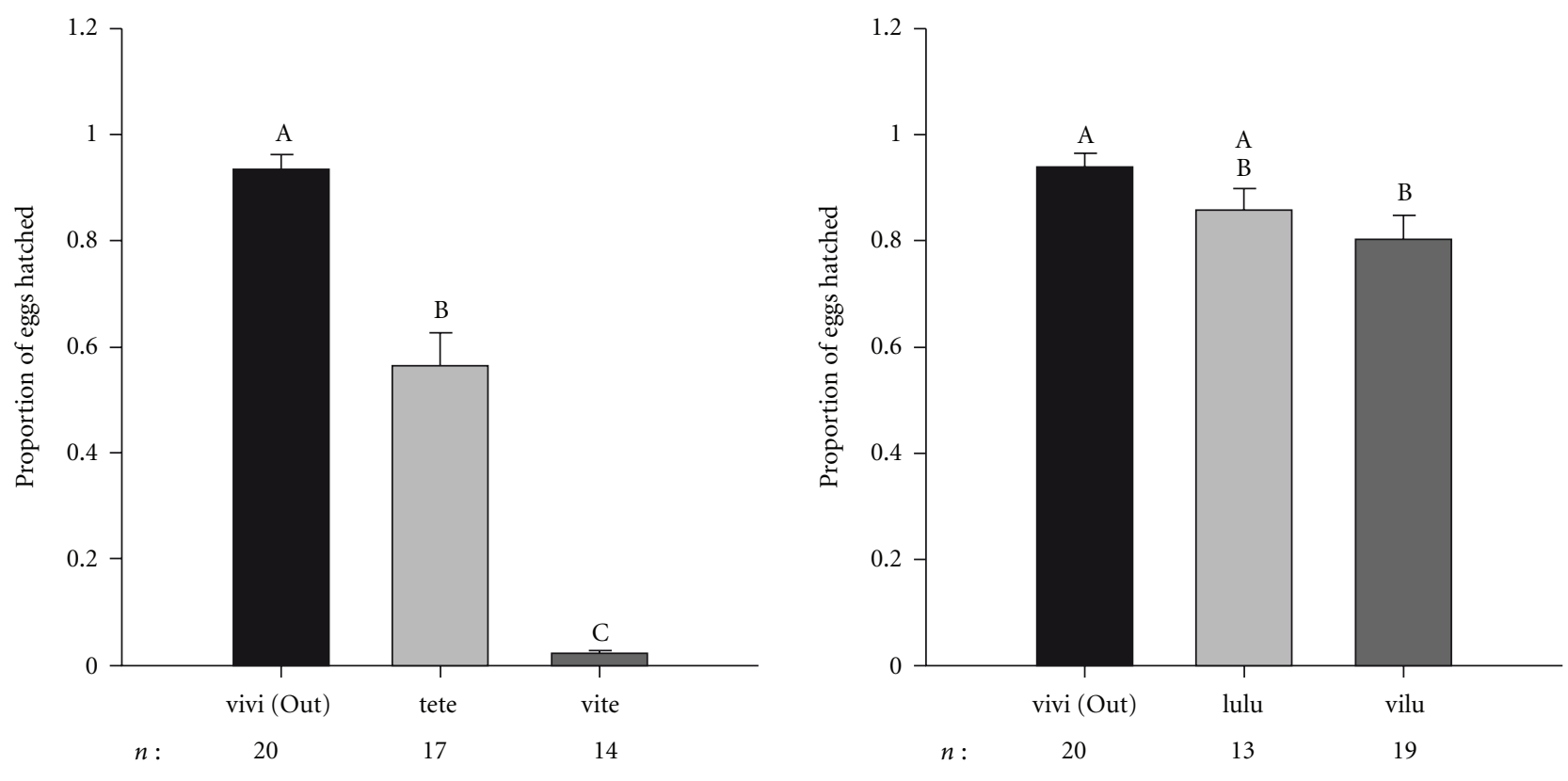

(b)

Figure 5: Average number of laid eggs and associated standard error for crosses involving D. virilis females and either $D$. a. texana (te) or $D$. lummei (lu) males (a). Average proportion of hatched eggs and associated standard error for crosses involving D. virilis females and either D. a. texana (te) or D. lummei (lu) males (b). Other labels are as in Figures 1 and 2.

a wide variety of egg-stimulating signals within the ejaculate. However, not all females within the species subgroup have retained this form of cryptic control. Preliminary data suggests that in crosses involving $D$. lummei, females respond by laying significantly higher number of eggs than in conspecific crosses (supplementary Figure 1S and supplementary Tables $1 \mathrm{~S}$ and $2 \mathrm{~S}$ in supplementary material available online at doi: $10.4061 / 2011 / 485460)$. This is a pattern more likely expected when males can overtake female resources to their advantage.
Overall, it appears that complex male-female interactions likely drive the evolution of egg laying phenotypes among species within the virilis subgroup.

Heterospecific crosses, with the exception of the cross between $D$. virilis females and D. lummei males, fail to fertilize enough eggs. Detailed studies done in D. melanogaster have characterized male ejaculate components that are known to influence the ability of sperm to properly store within female storage organs [28-33]. We also know that 
female secretions from the spemathecae and parovaria are required by sperm to fertilize the eggs [34-36], but it is unclear whether they affect sperm viability, sperm retention, or release from female storage. Our data from crosses between $D$. virilis females and D. novamexicana males shows that sperm is normally transferred but rapidly lost and such depletion of sperm could contribute to low egg fertilization. We do not know whether females actively dump or simply lose sperm during egg laying. Given the time frame at which sperm are being lost, and based solely on data from studies using $D$. melanogaster $[37,38]$, it seems unlikely that sperm is ejected by females but rather lost during the egg-laying process.

The production of similar numbers of eggs by females mated to heterospecific males when compared to conspecific crosses, and the fact that most eggs fail to hatch is likely costly as it represents an energetic burden for females to lay a large number of unfertilized eggs. However, rapid loss of sperm from the female storage organs after mating might explain why there has not been strong selection against the high egglaying phenotype. A female mating to a heterospecific male could actively dump or simply lose sperm from storage and become available and receptive to another mate. There is, in fact, evidence that $D$. melanogaster females with less sperm in storage are more likely to remate [39] and that female remating, in at least another Drosophila species, increases when a male is sperm depleted [40].

The PPI barrier among species within the subgroup is strong (Figures 2, 5 and 1S). Depending on the heterospecific cross performed, between approximately $75 \%$ and $98 \%$ of eggs failed to hatch. The cross between $D$. virilis females and D. lummei males was the only one for which we did not detect any form of PPI (see supplementary Table 2S). Drosophila virilis and D. lummei are the basal palearctic species to the subgroup, with chromosomal changes arisen during dispersal of the most recently derived species of $D$. a. americana, D. a. texana, and D. novamexicana (the novamexicana-americana clade) into North America [11]. Based on molecular data, D. virilis and D. lummei consistently appear as ancestral to species of the novamexicanaamericana clade, with $D$. virilis being the oldest species followed by $D$. lummei $[41,42]$. Moreover, estimated times of divergence show D. virilis arose about 6.5 Mya, closely followed by D. lummei (approximately $5.5 \mathrm{Mya}$ ) and a much later diversification of the novamexicana-americana clade around $1 \mathrm{Mya}$ [41]. The available phylogenetic information therefore supports $D$. virilis and D. lummei as ancestral species and more closely related to each other than to the other three species in the subgroup. Our results support that PPI due to low egg hatchability most likely evolved during the diversification of the novamexicana-americana clade from the two basal species of the virilis subgroup. Interestingly, PPI also appears to be a strong form of isolation among species within the most recently derived clade (novamexicanaamericana).

Finally, we know that $D$. virilis males show strong premating isolation, but $D$. virilis females readily mate with heterospecifics $[11,12]$. Coyne and Orr $[43,44]$ combined information on phylogenetic divergence and strength of premating and postzygotic isolation in the genus Drosophila to conclude that premating isolating barriers evolve earlier than other forms of isolation between diverging populations. It is then puzzling why $D$. virilis females do not show strong premating isolation with other species of the virilis subgroup. One possibility is that ordering isolating barriers by time of divergence (as in $[43,44]$ ) is not fully informative of their actual contribution to isolation as one cannot assume total independence among isolation mechanisms. Premating isolation might, therefore, not necessarily be the first barrier to hybridization. The other possibility might be that premating behavioural isolation between males of the ancestral species (D. virilis) and derived female species resulted from the evolution of polymorphism in receptors of derived male species to detect both short ancestral (D. virilis) and long derived species female cuticular hydrocarbons [45]. If so, the monomorphic male receptors in $D$. virilis males might not be able to detect heterospecific females as suitable mates [12].

In summary, our results demonstrate that $D$. virilis females exert control over egg-laying rates after mating and that PPI due to problems with egg hatching evolved as a strong reproductive isolating barrier among some of the species of the virilis subgroup. Our data from crosses with $D$. novamexicana males and data from earlier studies using $D$. a. americana and D. a. texana males indicate that PPI is due to different problems faced by the heterospecific sperm in female storage. It is yet unclear to what extent interspecies divergence of proteins of the male ejaculate and/or female reproductive tract secretions might contribute to heterospecific sperm problems in the $D$. virilis female reproductive tract.

\section{Acknowledgments}

The authors would like to thank Jens Franck and two anonymous referees for their comments and suggestions. This work was funded by an NSERC Discovery Grant to A. Civetta. N. Sagga was fully funded by a graduate scholarship from the Government of Saudi Arabia.

\section{References}

[1] J. A. Coyne and H. A. Orr, Speciation, Sinauer Associates, Sunderland, Mass, USA, 2004.

[2] P. G. Gregory and D. J. Howard, "A post-insemination barrier to fertilization isolates two closely related ground crickets," Evolution, vol. 48, no. 3, pp. 705-710, 1994.

[3] C. S. C. Price, "Conspecific sperm precedence in Drosophila," Nature, vol. 388, no. 6643, pp. 663-666, 1997.

[4] D. J. Howard, P. G. Gregory, J. Chu, and M. L. Cain, "Conspecific sperm precedence is an effective barrier to hybridization between closely related species," Evolution, vol. 52, no. 2, pp. 511-516, 1998.

[5] C. S.C. Price, C. H. Kim, C. J. Gronlund, and J. A. Coyne, "Cryptic reproductive isolation in the Drosophila simulans species complex," Evolution, vol. 55, no. 1, pp. 81-92, 2001.

[6] L. W. Simmons, Sperm Competition and Its Evolutionary Consequences in the Insects, Princeton University Press, Princeton, NJ, USA, 2001. 
[7] N. Sellier, J. M. Brun, M. M. Richard, F. Batellier, V. Dupuy, and J. P. Brillard, "Comparison of fertility and embryo mortality following artificial insemination of common duck females (Anas Platyrhynchos) with semen from common or Muscovy (Cairina Moschata) drakes," Theriogenology, vol. 64, no. 2, pp. 429-439, 2005.

[8] T. R. Birkhead and J. P. Brillard, "Reproductive isolation in birds: postcopulatory prezygotic barriers," Trends in Ecology and Evolution, vol. 22, no. 5, pp. 266-272, 2007.

[9] E. S. Kelleher and T. A. Markow, "Reproductive tract interactions contribute to isolation in Drosophila," Fly, vol. 1, no. 1, pp. 33-37, 2007.

[10] D. R. Matute and J. A. Coyne, "Intrinsic reproductive isolation between two sister species of Drosophila," Evolution, vol. 64, no. 4, pp. 903-920, 2010.

[11] L. H. Throckmorton, "The virilis species group," in The Genetics and Biology of Drosophila, M. Ashburner, H. L. Carson, and J. N. Thompson Jr., Eds., vol. 3b, pp. 227-296, Academic Press, London, UK, 1982.

[12] D. Nickel and A. Civetta, "An X chromosome effect responsible for asymmetric reproductive isolation between male Drosophila virilis and heterospecific females," Genome, vol. 52, no. 1, pp. 49-56, 2009.

[13] H. A. Orr and J. A. Coyne, "The genetics of postzygotic isolation in the Drosophila virilis group," Genetics, vol. 121, no. 3, pp. 527-537, 1989.

[14] J. Lumme and E. Heikkinen, "Viability of first and second generation hybrids of Drosophila virilis and Drosophila lummei," Heredity, vol. 65, pp. 435-447, 1990.

[15] K. Lamnissou, M. Loukas, and E. Zouros, "Incompatibilities between Y chromosome and autosomes are responsible for male hybrid sterility in crosses between Drosophila virilis and Drosophila texana," Heredity, vol. 76, p. 6, 1996.

[16] A. L. Sweigart, "The genetics of postmating, prezygotic reproductive isolation between Drosophila virilis and D. americana," Genetics, vol. 184, no. 2, pp. 401-410, 2010.

[17] J. T. Patterson and W. S. Stone, Evolution in the Genus Drosophila, Macmillan and Co., New York, NY, USA, 1952.

[18] T. A. Markow and P. M. O'Grady, "Drosophila biology in the genomic age," Genetics, vol. 177, no. 3, pp. 1269-1276, 2007.

[19] R. M. Warn and A. Warn, "Microtubule arrays present during the syncytial and cellular blastoderm stages of the early Drosophilia embryo," Experimental Cell Research, vol. 163, no. 1, pp. 201-210, 1986.

[20] M. F. Wolfner, "Battle and ballet: molecular interactions between the sexes in drosophila," Journal of Heredity, vol. 100, no. 4, pp. 399-410, 2009.

[21] M. Soller, M. Bownes, and E. Kubli, "Mating and sex peptide stimulate the accumulation of yolk in oocytes of Droosophila melanogaster," European Journal of Biochemistry, vol. 243, no. 3, pp. 732-738, 1997.

[22] M. Soller, M. Bownes, and E. Kubli, "Control of oocyte maturation in sexually mature Drosophila females," Developmental Biology, vol. 208, no. 2, pp. 337-351, 1999.

[23] S. A. Monsma and M. F. Wolfner, "Structure and expression of a Drosophila male accessory gland gene whose product resembles a peptide pheromone precursor," Genes \& Development, vol. 2, no. 9, pp. 1063-1073, 1988.

[24] Y. Heifetz, O. Lung, E. A. Frongillo, and M. F. Wolfner, "The Drosophila seminal fluid protein Acp26Aa stimulates release of oocytes by the ovary," Current Biology, vol. 10, no. 2, pp. 99-102, 2000.
[25] D. J. Begun and H. A. Lindfors, "Rapid evolution of genomic Acp complement in the melanogaster subgroup of Drosophila," Molecular Biology and Evolution, vol. 22, no. 10, pp. 20102021, 2005.

[26] D. J. Begun, H. A. Lindfors, M. E. Thompson, and A. K. Holloway, "Recently evolved genes identified from Drosophila yakuba and D. erecta accessory gland expressed sequence tags," Genetics, vol. 172, no. 3, pp. 1675-1681, 2006.

[27] W. Haerty, S. Jagadeeshan, R. J. Kulathinal et al., "Evolution in the fast lane: rapidly evolving sex-related genes in Drosophila," Genetics, vol. 177, no. 3, pp. 1321-1335, 2007.

[28] A. Wong, S. N. Albright, J. D. Giebel et al., "A role for Acp29AB, a predicted seminal fluid lectin, in female sperm storage in Drosophila melanogaster," Genetics, vol. 180, no. 2, pp. 921-931, 2008.

[29] D. M. Neubaum and M. F. Wolfner, "Mated Drosophila melanogaster females require a seminal fluid protein, Acp36DE, to store sperm efficiently," Genetics, vol. 153, no. 2, pp. 845-857, 1999.

[30] T. Chapman, D. M. Neubaum, M. F. Wolfner, and L. Partridge, "The role of male accessory gland protein Acp36DE in sperm competition in Drosophila melanogaster," Proceedings of the Royal Society Biological Sciences, vol. 267, no. 1448, pp. 10971105, 2000.

[31] M. J. Bertram, D. M. Neubaum, and M. F. Wolfner, "Localization of the Drosophila male accessory gland protein Acp36DE in the mated female suggests a role in sperm storage," Insect Biochemistry and Molecular Biology, vol. 26, no. 8-9, pp. 971980, 1996.

[32] F. W. Avila and M. F. Wolfner, "Acp36DE is required for uterine conformational changes in mated Drosophila females," Proceedings of the National Academy of Sciences of the United States of America, vol. 106, no. 37, pp. 15796-15800, 2009.

[33] J. L. Mueller, J. R. Linklater, K. R. Ram, T. Chapman, and M. F. Wolfner, "Targeted gene deletion and phenotypic analysis of the Drosophila melanogaster seminal fluid protease inhibitor Acp62F," Genetics, vol. 178, no. 3, pp. 1605-1614, 2008.

[34] R. C. Anderson, "A study of the factors affecting fertility of lozenge females of Drosophila melanogaster," Genetics, vol. 30, pp. 280-296, 1945.

[35] A. K. Allen and A. C. Spradling, "The Sf1-related nuclear hormone receptor $\mathrm{Hr} 39$ regulates Drosophila female reproductive tract development and function," Development, vol. 135, no. 2 , pp. 311-321, 2008.

[36] A. Prokupek, F. Hoffmann, S. I. Eyun, E. Moriyama, M. Zhou, and L. Harshman, "An evolutionary expressed sequence tag analysis of drosophila spermatheca genes," Evolution, vol. 62, no. 11, pp. 2936-2947, 2008.

[37] R. R. Snook and D. J. Hosken, "Sperm death and dumping in Drosophila," Nature, vol. 428, no. 6986, pp. 939-941, 2004.

[38] M. K. Manier, J. M. Belote, K. S. Berben, D. Novikov, W. T. Stuart, and S. Pitnick, "Resolving mechanisms of competitive fertilization success in Drosophila melanogaster," Science, vol. 328, no. 5976, pp. 354-357, 2010.

[39] M. E. A. Newport and M. H. Gromko, "The effect of experimental design on female receptivity to remating and its impact on reproductive success in Drosophila melanogaster," Evolution, vol. 38, pp. 1261-1272, 1984.

[40] N. Luck and D. Joly, "Sexual selection and mating advantages in the giant sperm species, Drosophila bifurca," Journal of Insect Science, vol. 5, p. 10, 2005. 
[41] G. S. Spicer and C. D. Bell, "Molecular phylogeny of the Drosophila virilis species group (Diptera: Drosophilidae) inferred from mitochondrial $12 \mathrm{~S}$ and $16 \mathrm{~S}$ ribosomal RNA genes," Annals of the Entomological Society of America, vol. 95, no. 2, pp. 156-161, 2002.

[42] B. C. Caletka and B. F. McAllister, "A genealogical view of chromosomal evolution and species delimitation in the Drosophila virilis species subgroup," Molecular Phylogenetics and Evolution, vol. 33, no. 3, pp. 664-670, 2004.

[43] J. A. Coyne and H. A. Orr, "Patterns of speciation," Evolution, vol. 43, pp. 362-381, 1989.

[44] J. A. Coyne and H. A. Orr, "Patterns of speciation in Drosophila," Evolution, vol. 52, pp. 295-303, 1997.

[45] R. J. Bartelt, M. T. Armold, A. M. Schaner, and L. L. Jackson, "Comparative analysis of cuticular hydrocarbons in the Drosophila virilis species group," Comparative Biochemistry and Physiology-Part B: Biochemistry and, vol. 83, no. 4, pp. 731-742, 1986. 

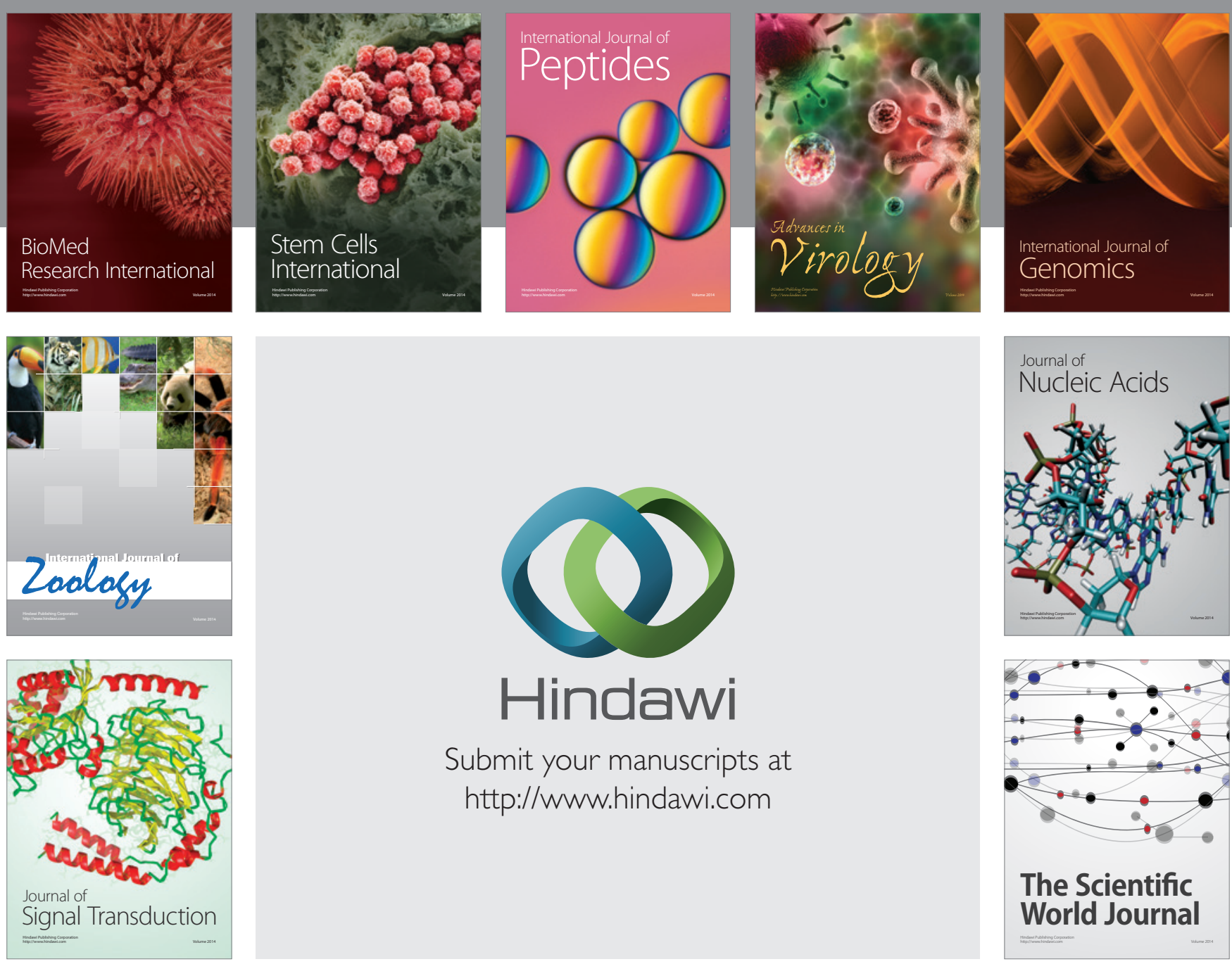

Submit your manuscripts at

http://www.hindawi.com
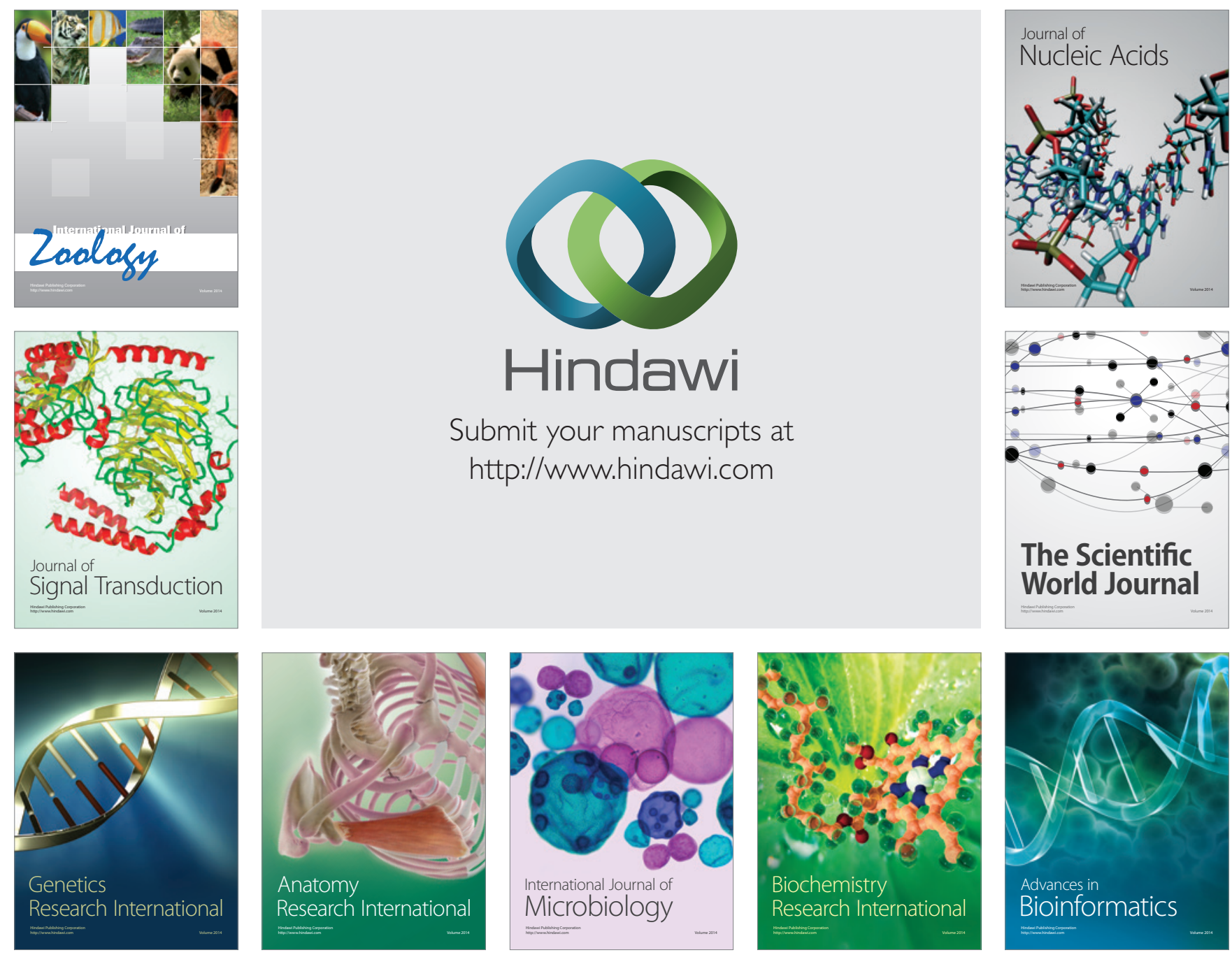

The Scientific World Journal
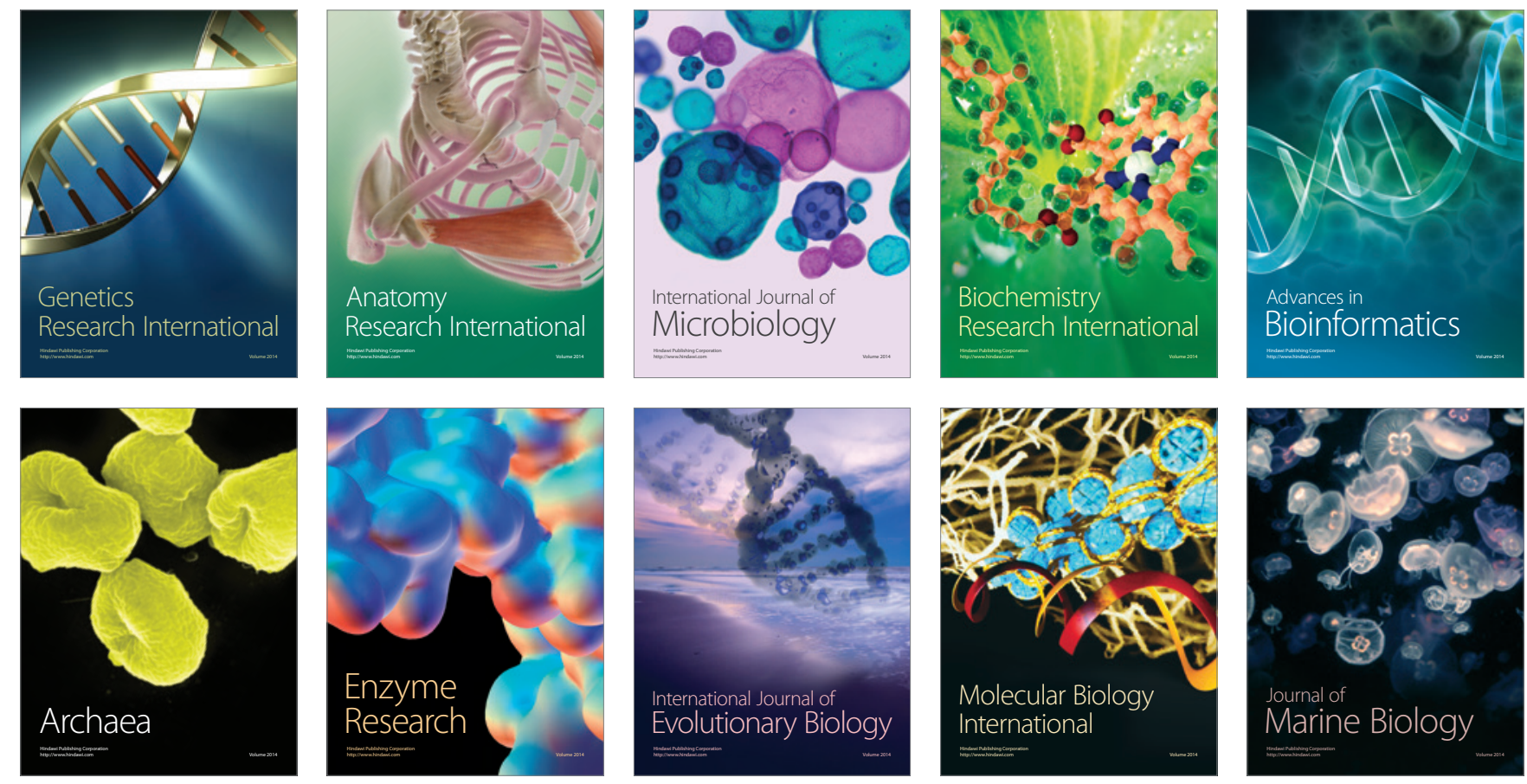